\title{
On the Borel Summability of Planar Perturbation Series: Transition to Minkowski Space
}

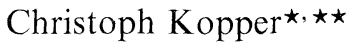

Institute for Theoretical Physics, Princetonplein 6, P.O. Box 80.006, NL-3508 TA Utrecht, The Netherlands

\begin{abstract}
In recent years ' $t$ Hooft and Rivasseau proved the Borel summability of planar asymptotically free massive theories in Euclidean space. The corresponding Borel sums in Minkowski space are shown to exist as linear functionals if the Euclidean counterparts are bounded polynomially in momentum space and fulfill certain analyticity conditions. Both can be verified in massive planar "wrong sign" $\varphi_{4}^{4}$ using Rivasseau's approach. The functionals alternatively are densely defined and unbounded on an $L^{p}$ space or bounded on (the whole of) a Banach space with a more restrictive norm.
\end{abstract}

\section{Introduction}

A few years ago 't Hooft $[1,2]$ and Rivasseau [3] established the Borel summability of planar asymptotically free theories without any massless particles in four-dimensional Euclidean space. We shall try to translate those results to Minkowski space.

Our work is based on the methods of Rivasseau which explicitly display the relation between the renormalized perturbative series for a given one particle irreducible (1PI) Green function and its Borel sum which is shown to be identical to a sum over certain dressed Feynman amplitudes. He restricts to a $U(N)$ [or $O(N)]$ symmetric massive scalar matrix field theory with the sign of the scalar selfcoupling chosen such that the theory becomes asymptotically free. (The case where the scalar fields are in the fundamental $N$-vector representation is far simpler. The limit $N \rightarrow \infty$ can even be obtained analytically, see e.g. [4].) In principle this method should be applicable to more general examples as regarded by 't Hooft $[1,2]$, i.e. planar gauge theories where all particles become massive through the Higgs mechanism and all couplings asymptotically free on imposing suitable

\footnotetext{
* Supported by a scholarship of the NATO scientific committee via the German academic exchange service (DAAD)

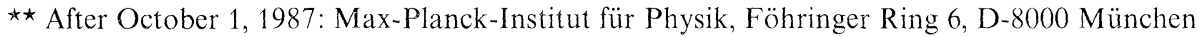
40, Federal Republic of Germany
} 
boundary conditions for the renormalization group equations. But the technical complications would be considerable.

Quite generally Green functions in Minkowski space are known to exist only as linear functionals on certain test function spaces due to their singularities. Mostly they are interpreted as tempered distributions. We are not able to prove the existence of the Borel sums of planar $\varphi_{4}^{4}$ Green functions as tempered distributions because we don't have sufficient understanding of the singularity structure of all possible planar diagrams, but we can show that these Borel sums exist as linear functionals on a suitable test function space. With respect to the latter we can take two attitudes: Either we choose (a dense subspace of) an $L^{p}$-space (in the external momentum variables) and get a densely defined unbounded functional, or we choose a space of functions with a much more restrictive norm and get a bounded functional on the whole of this space. Since one may take the same attitude with respect to tempered distributions, the interpretation we shall give to the Borel sums of Green functionals is not more restrictive but rather complementary to the standard approach.

The general attitude towards the transition to Minkowski space in constructive field theory is determined by the belief that for a reasonable physical model - if constructed in the Euclidean - it should be possible to verify the Osterwalder Schrader (OS) axioms [5] guaranteeing the existence of a Wightman theory in Minkowski space. Several reasons may call for complementary procedures. There may be reasonable physical models which do not fulfill all the axioms such as planar "wrong sign" $\varphi_{4}^{4}$ which presumably has no true, but an infinitely long-lived metastable ground state [6] and probably violates OS positivity. Also the OS procedure might obscure the persistence of certain properties of Euclidean quantities in Minkowski space, in the present case the asymptotic character of the perturbation expansion which still seems to be the strongest argument for the belief in quantum field theory quite generally.

The method to be presented is general enough to deduce the existence of Minkowski space quantities in other cases under certain conditions on the Euclidean counterparts. Euclidean quantities in momentum space should be polynomially bounded, and the modifications of bare perturbation theory through dressing factors should, roughly speaking, not introduce additional singularities in those parts of the complex momentum plane which are sweeped out by Wick rotations.

Section II of this paper presents a short review of Rivasseau's results and of those ingredients of his procedure we shall need later on. In Sect. III we construct the related Minkowski space functionals starting from regularized dressed Minkowski amplitudes. The essential tool for this construction are sets of functions in the Hilbert space $L^{2}([0, \infty))$ with certain analyticity and invariance properties in the upper right complex plane. Some of the information on those functions is presented in the appendix. We end with a short summary and discussion of the results.

\section{Planar $\varphi_{4}^{\mathbf{4}}$. Results in Euclidean Space}

We present a short review of Rivasseau's results [3] in Euclidean space which in turn largely depend on an earlier paper by de Calan and Rivasseau [7]. We 
consider a massive Hermitian $N \times N$-matrix field $\varphi$ in four-dimensional space. The Lagrangian

$$
\mathscr{L}=\operatorname{tr}\left\{\frac{1}{2}\left(\partial_{\mu} \varphi\right)^{2}-\frac{m^{2}}{2} \varphi^{2}+g \varphi^{4}\right\}
$$

is invariant under the transformation

$$
\varphi \rightarrow U^{-1} \varphi U \text { for any } U(N) \text { Matrix } U \text {. }
$$

$g>0$ corresponds to the asymptotically free side of the theory. The connected Schwinger functions can be derived from $\mathscr{L}$ via the standard formal procedure. They do not make sense without renormalization. In a massive theory we can choose the BPHZ renormalization scheme and perform the necessary subtractions at 0 external momenta. Then after rescaling the coupling $(g \rightarrow g / N)$ the perturbative expansion in $g$ of any $U(N)$ invariant Schwinger function is dominated in the limit $N \rightarrow \infty$ by the planar diagrams [8]. The restriction to planar diagrams is crucial for Borel summability: The number $A$ of (topologically distinct) Feynman diagrams with $L$ loops contributing to a given Schwinger function is bounded in the planar case by $[1,9]$

$$
A \leqq K_{E} C^{L}
$$

( $C$ is of order $10, K_{E}$ grows with the number of external lines $E$ ) whereas it grows factorially if one considers all diagrams at a given order [7]. Since diagrams with $L$ loops carry a factor $g^{L}$, the perturbation expansion would even be absolutely convergent for sufficiently small $g$ were it not for the unlimited growth of certain individual Feynman diagram contributions in high orders $[10,11]$. The kind of singularity involved here was called renormalon by " $t$ Hooft because it is related to the necessity of performing more and more renormalization operations on those diagrams, and it was his early suspicion that renormalons can be eliminated through asymptotic freedom in massive theories [11]. The concept was elaborated on further by Parisi [12].

The connected 1PI Schwinger functions of planar $\varphi_{4}^{4}$ with $E$ external momenta are given by the formal power expansion

$$
\delta\left(\sum_{q=1}^{E} p_{q}\right) S_{E}(p ; g)=\delta\left(\sum_{q=1}^{E} p_{q}\right) \sum_{n=0}^{\infty} g^{n} a_{n}^{E}(p) .
$$

$p=\left(p_{1}, \ldots, p_{E}\right)$ is a set of external 4-momenta and $a_{n}^{E}$ is the sum over all renormalized Feynman-amplitudes associated to (topologically distinct) 1PI Feynman graphs with $n$ internal vertices and $E$ external legs. The Feynman amplitude for a given graph $G$ in momentum space, renormalized according to Zimmermann's forest formula [13] as translated to $\alpha$ parametric space [14] is given by

$$
\begin{gathered}
I_{G}(p)=\int_{0}^{\infty} \ldots \int_{0}^{\infty} \prod_{i=1}^{l} d \alpha_{i} \exp \left\{-m^{2} \sum_{i=1}^{l} \alpha_{i}\right\} \mathscr{R} Z_{G}(p, \alpha), \\
Z_{G}(p, \alpha)=\frac{1}{U_{G}^{2}(\alpha)} \exp \left\{-V_{G}(p, \alpha) / U_{G}(\alpha)\right\}, \\
U_{G}(\alpha)=\sum_{S}\left(\prod_{i \notin S} \alpha_{i}\right), \quad V_{G}(p, \alpha)=\sum_{T}\left(\prod_{i \notin T} \alpha_{i}\right)\left(\sum_{i \in E_{1}} p_{q}\right)^{2}, \\
\mathscr{R}=\sum_{\mathscr{F}} T_{\mathscr{F}}, \quad T_{\mathscr{F}}=\prod_{F \in \mathscr{F}}\left(-t_{F}\right) .
\end{gathered}
$$


$U, V$ are the Symanzik polynomials of $G$ (see e.g. [15]). $S$ runs over the (one-)trees of $G, T$ over the "two-trees" (one-trees minus one line). $E_{1}$ is one of the two sets of external momenta into which $G$ is divided by the two-tree (no matter which). The renormalization operator $\mathscr{R}$ contains a sum over all (closed divergent) forests $\mathscr{F}$ of renormalization parts of $G$. For any forest $\mathscr{F}$ (a partially ordered set of divergent subdiagrams $F$ ) a product of Taylor operations $t_{F}$ acting on $Z$ in parametric space is introduced which lead to an elimination of the divergences coming from the subdiagrams in the final expression (4). The ultraviolet divergences in momentum space are reflected by divergences in parametric space for certain $\alpha$-parameters going to 0 . A more explicit form of (4) (as well as many more explanations) can be found in [7], especially Lemma III.4 (err.).

Before stating Rivasseau's results we have to introduce his dressing factors. We cannot give any details here, but should mention that they are a result of a partial resummation of perturbation theory: at any vertex and also at certain "reduction vertices" asymptotic freedom is taken into account by iteratively inserting the 1-loop-contribution to the four-point function. Rivasseau proves this modification to be sufficient to eat up the renormalons on one side and not too drastic to prevent the unmodified series from being asymptotic to the modified one on the other side. We define

$$
\begin{gathered}
\xi(x)=\int_{x}^{\infty} \frac{d \alpha}{\alpha} \int_{0}^{1} \exp \left\{-m^{2} \alpha(1+\beta)\right\}(1+\beta)^{-2} 2 d \beta, \quad x>0, \\
\operatorname{od}(x, g)=(1+8 g \xi(x))^{-1} \quad \text { (od from “ordinary dressing”). }
\end{gathered}
$$

For $x \rightarrow 0 \xi(x)=O(|\log x|)$ so that od $(x, g)$ vanishes logarithmically. Note that od is singular for $g<0$ (i.e. no asymptotic freedom),

$$
\zeta(x, y ; g)=\int_{x}^{y} \frac{d \alpha}{\alpha} \frac{g}{1+8 g \xi(\alpha)} \int_{0}^{1} \exp \left\{-m^{2} \alpha(1+\beta)\right\}(1+\beta)^{-2} 2 d \beta, \quad 0<x \leqq y .
$$

Bounds on $\zeta, \xi$ are given by Rivasseau [3, Lemmata A.2 and A.4]. The dressing factors are then of the form

$$
D_{G}^{\mathscr{F}}(\alpha, g)=\prod_{j=1}^{n} \operatorname{od}\left(\alpha_{j}, g\right) \prod_{h=1}^{s} \exp \left\{-16 \zeta\left(\tilde{\alpha}_{h}, \alpha_{h} ; g\right)\right\}, \quad \tilde{\alpha}_{h} \leqq \alpha_{h} .
$$

$n$ is the number of vertices of $G$; for a given forest $\mathscr{F} s$ is a certain subset of the subgraphs of $G$ in $\mathscr{F}$ with four external legs. This subset as well as the arguments of od and $\zeta$ in (11) depend on the ordering of the $\alpha$-parameters in (4), i.e. on the respective Hepp sector $H_{\sigma}$

$$
H_{\sigma}=\left\{\left(\alpha_{1} \ldots \alpha_{l}\right) \mid 0 \leqq \alpha_{\sigma(1)} \leqq \ldots \leqq \alpha_{\sigma(l)}<\infty\right\}, \quad \sigma \text { a permutation of }\{1, \ldots, l\} .
$$

We thus get the following expression for the dressed renormalized amplitude

$$
I_{G, d r}(p, g)=\int_{0}^{\infty} \cdots \int_{0}^{\infty} \prod_{i=1}^{l} d \alpha_{i} \exp \left\{-m^{2} \sum_{i=1}^{l} \alpha_{i}\right\} \sum_{\mathscr{F}}^{\prime} D_{G}^{\mathscr{F}}(\alpha, g) \prod_{F \in \mathscr{F}}\left(-t_{F}\right) Z_{G}(p, \alpha),
$$

where $\sum_{\mathscr{F}}^{\prime}$ as compared to $\sum_{\mathscr{\mathscr { F }}}$ in $(7)$ contains restrictions depending on the Hepp sectors. These restrictions are a remnant of the fact that the corresponding missing counterterms have been resummed to create the dressing factors. 
Now we can state Rivasseau's results on planar $\varphi_{4}^{4}$-amplitudes in Euclidean space.

R1 The sum over all dressed amplitudes of 1PI graphs with $E$ external legs $\sum_{G} g^{n(G)} I_{G, d r}(p, g)$ is absolutely convergent for complex $g$ with Reg $>0,|g|<\eta, \eta$ sufficiently small (s.s.). This sum, called $S_{E}(p, g)$, is analytic in that domain.

R2 (Borel summability). There exists a constant $K$ such that for Reg $>0,|g|<\eta$ (s.s.) one has for any $k \in \mathbb{N}$,

$$
\left|S_{E}(p, g)-\sum_{G / n(G) \leqq k-1} g^{n(G)} I_{G}(p)\right| \leqq k !(K|g|)^{k} .
$$

R3 For any graph $G$ of order $n$, planar or not, the dressed amplitude is bounded for $\operatorname{Reg}>0,|g|<\eta$ (s.s.) by

$$
\begin{aligned}
\left|g^{n} I_{G . d r}(p, g)\right| \leqq\left\{\begin{array}{ll}
K_{E}^{n}(\eta) & \text { if } \quad E \geqq 4, \\
\left(p^{2}+m^{2}\right) K_{2}^{n}(\eta) & \text { if } \quad E=2,
\end{array} \quad K_{E}(\eta)\right. \text { fixed, } \\
K_{E}(\eta) \rightarrow 0 \\
\eta \rightarrow 0
\end{aligned}
$$

As remarked by Rivasseau it is possible - at the cost of a smaller $\eta$ - to allow for $|\arg g|<\pi-\delta, \delta>0$ instead of $\operatorname{Reg}>0$. In this case $\eta \rightarrow 0$ for $\delta \rightarrow 0$. Thus only negative $g$ are strictly excluded.

\section{Transition to Minkowski Space}

The (dressed) amplitude associated with a given 1PI graph in Minkowski space only makes sense as a linear functional, e.g. a tempered distribution, on a space of test functions. To actually calculate such an expression one has to introduce a regularization parameter $\varepsilon$ (Feynman- $\varepsilon$ ) which implies a prescription on how to treat the poles of the propagators. As emphasized by Zimmermann the standard way of doing this is insufficient from the mathematical point of view because it still leaves us with only conditionally convergent integrals (even if renormalized) in Minkowski space [16]. We thus adopt his way of regularization which he has shown to meet with all mathematical and physical requirements.

The regularized propagator is taken to be

$$
\frac{1}{p_{0}^{2}-\mathbf{p}^{2}-m^{2}+i \varepsilon\left(\mathbf{p}^{2}+m^{2}\right)},
$$

so that

$$
\left|\frac{1}{p_{0}^{2}-\mathbf{p}^{2}-m^{2}+i \varepsilon\left(\mathbf{p}^{2}+m^{2}\right)}\right| \leqq\left(\frac{1}{\varepsilon}+\left(1+\frac{1}{\varepsilon^{2}}\right)^{1 / 2}\right) \frac{1}{p_{0}^{2}+\mathbf{p}^{2}+m^{2}} .
$$

Thus for finite $\varepsilon$ any Feynman-amplitude can be estimated in terms of its Euclidean counterpart and inverse powers of $\varepsilon$. Writing

$$
\frac{i}{p_{0}^{2}-\mathbf{p}^{2}-m^{2}+i \varepsilon\left(\mathbf{p}^{2}+m^{2}\right)}=\int_{0}^{\infty} d \alpha e^{i \alpha\left[p_{0}^{2}-\mathbf{p}^{2}-m^{2}+i \varepsilon\left(\mathbf{p}^{2}+m^{2}\right)\right]}
$$


we can represent the dressed renormalization Feynman amplitude for a graph $G$ in parametric space. The expression corresponding to (12) is

$$
I_{G, \varepsilon}(p, g)=\int_{0}^{\infty} \ldots \int_{0}^{\infty} \prod_{i=1}^{l} d a_{i} e^{-i \Sigma \alpha_{i}(1-i \varepsilon)} \sum_{\mathscr{F}}^{\prime} \hat{D}_{G}^{\mathscr{F}}(\alpha, \varepsilon, g) \prod_{F \in \mathscr{F}}\left(-t_{F}\right) \hat{Z}_{G}(p, \alpha, \varepsilon) .
$$

In (16) - and all subsequent formulae - we have chosen $m^{2}=1$, i.e. we measure all momenta in units of $m$. [A factor $(-i)^{L}(1-i \varepsilon)^{\frac{3 L}{2}}$ with $L$ the number of loops of $G$ has been absorbed in $\left.I_{G, \varepsilon}\right]$,

$$
\hat{Z}_{G}(p, \alpha, \varepsilon)=\frac{1}{U_{G}^{2}(\alpha)} \exp \left\{i \frac{V_{G}\left(p_{0}, \alpha\right)}{U_{G}(\alpha)}-i \frac{V_{G}(\mathbf{p}, \alpha)}{U_{G}(\alpha)}(1-i \varepsilon)\right\} .
$$

The Minkowski space dressing factors $\hat{D}$ are derived from the Euclidean ones by substituting for (8)-(10)

$$
\begin{gathered}
\hat{\xi}_{\varepsilon}(x)=\int_{x}^{\infty} \frac{d \alpha}{\alpha} \int_{0}^{1} e^{-i \alpha(1+\beta)(1-i \varepsilon)} 2(1+\beta)^{-2} d \beta \\
=\int_{0}^{\infty} \frac{d \alpha}{\alpha+i x} \int_{0}^{1} e^{-(\alpha+i x)(1+\beta)(1-i \varepsilon)} 2(1+\beta)^{-2} d \beta, \\
\hat{\zeta}_{\varepsilon}(x, y, g)=\int_{x}^{y} \frac{d \alpha}{\alpha} \frac{g}{1+8 g \hat{\xi}_{\varepsilon}(\alpha)} \int_{0}^{1} e^{-i \alpha(1+\beta)(1-i \varepsilon)} 2(1+\beta)^{-2} d \beta .
\end{gathered}
$$

It is easy to see from these expressions that by analytic continuation and for $\operatorname{Reg}>0,|g|$ s.s.

$$
\begin{aligned}
& \lim _{\varepsilon \rightarrow 0}\left(\hat{o d}_{\varepsilon}(-i x ; g), \exp \left\{-\hat{\zeta}_{\varepsilon}(-i x,-i y ; g)\right\}\right) \\
& \quad=(\operatorname{od}(x ; g), \exp \{-\zeta(x, y ; g)\}) \quad(0<x \leqq y)
\end{aligned}
$$

(where the bound on $|g|$ is far less restrictive than the one which was necessary in $\mathrm{R} 1), \ldots, \mathrm{R} 3)$ or even in $[1,2]$ ) and also that for those $g$ the integrand of $\hat{\zeta}$ is free from singularities because $\lim _{x \rightarrow 0}\left|\arg \hat{\xi}_{\varepsilon}(x)\right|=0$. For any such $g \quad \hat{o d}_{\varepsilon}(\lambda ; g)$, $\exp \left\{-\hat{\zeta}_{\varepsilon}(\lambda x, \lambda y ; g)\right\}$ are (in $x, y$ uniformly) bounded analytic functions of $\lambda$ in the lower right half plane $\backslash\{\lambda=0\}$ approaching 0 here for $|\lambda| \rightarrow 0$.

Lorentz invariance of the amplitudes is reflected by the fact that $V_{G}(p, \alpha)$ is a function of the Minkowski space squares $\left(\sum p_{q}\right)^{2}$ only (up to the $\varepsilon$-terms). The sum is here over some subset of the external momenta. It is the time-like part of the space of external momenta $\left\{p_{01}, \ldots, p_{0 E-1}\right\}$ we are especially interested in. Here we introduce polar coordinates,

$$
p_{01}=r \omega_{1}, \ldots, p_{0 E-1}=r \omega_{E-1}, \quad E>2,
$$

where $r=\left(p_{01}^{2}+\ldots+p_{0 E-1}^{2}\right)$ and $\omega_{i}$ depend on the angles only. We now want to apply $I_{G, \varepsilon}\left(r^{2}, \omega, \mathbf{p}\right)$ to test functions depending on $r$. (Of course we can smear out with respect to the other variables, too, but it is not necessary.) By Wick-rotation $r \rightarrow i r$, we then try to find our way back to the Euclidean results. The necessary 
restrictions on the test functions are shown still to allow for a set dense in $L^{1}([0, \infty), d r)$.

We define the linear space of functions $S$ by:

where

$$
\mathrm{S}=\{\varphi \mid \varphi:[0, \infty) \rightarrow \mathbb{C}, \varphi \text { fulfills S1) S2) }\}
$$

S1) $\varphi$ has an analytic continuation to the upper right complex half plane

$$
\left\{z \mid z=r e^{i \gamma}, 0<r<\infty, 0 \leqq \gamma \leqq \frac{\pi}{2}\right\}
$$

and is continuous (bounded) for $r \rightarrow 0$.

S2) $r^{n} \varphi\left(r e^{i \gamma}\right) \rightarrow 0$ for $r \rightarrow \infty$ and any $n \in \mathbb{N}$; uniformly in $\gamma \in\left[0, \frac{\pi}{2}\right]$.

Lemma 1. $S$ is dense in $L^{2}([0, \infty), d r)$.

Proof. Any function $P(r) e^{-\frac{1-i}{2} r}$ for any arbitrary polynomial $P(r)$ fulfills S1) S2).

So $S$ contains the Laguerre functions multiplied by the phase factor $e^{\frac{i}{2} r}$, i.e. a complete orthonormal system in $L^{2}([0, \infty), d r)$.

Furthermore we define

$$
\hat{S}=\left\{\hat{\varphi}|\hat{\varphi}:(-\infty, \infty) \rightarrow \mathbb{C}, \theta(r) \varphi(r)|_{\geqq} \in S,\left.\theta(-r) \varphi(r)\right|_{\geqq} \in S\right\}
$$

(here $\left.\right|_{\geqq}$indicates restriction to $[0, \infty)$.)

$\hat{S}$ is then obviously dense in $L^{2}((-\infty, \infty), d r)$.

For $\varphi \in \hat{S}$ and a contribution to the two-point function $(E=2) I_{G, \varepsilon}\left(p_{0}^{2}, \mathbf{p}^{2} ; g\right)$, we now write

$$
\int_{-\infty}^{\infty} d p_{0} I_{G, \varepsilon}\left(p_{0}^{2}, \mathbf{p}^{2} ; g\right) \hat{\varphi}\left(p_{0}\right)=\int_{0}^{\infty} d r I_{G, \varepsilon}\left(r^{2}, \mathbf{p}^{2} ; g\right) \varphi(r),
$$

where $\varphi(r)=\left(\left.\theta(r) \hat{\varphi}(r)\right|_{\geqq}+\left.\theta(-r) \hat{\varphi}(-r)\right|_{\geqq}\right) \in S$, thus unifying the cases $E>2$ and $E=2$.

For $\varphi \in S$ the integral over $I_{G, \varepsilon}\left(r^{2}, \omega, \mathbf{p} ; g\right) \varphi(r)$ is well-defined for $\varepsilon>0$ as a consequence of R3), the general estimate (14) and the definition of $S$. (At this stage we could have been less restrictive with respect to the behaviour of $\varphi(r), r \rightarrow \infty)$. We now use the definition of $I_{G, \varepsilon}(16)$ to get:

$$
\begin{aligned}
\int_{0}^{\infty} d r & I_{G, \varepsilon}\left(r^{2}, \omega, \mathbf{p} ; g\right) \varphi(r) \\
= & \int_{0}^{\infty} d r \varphi(r) \int_{0}^{\infty} \cdots \int_{0}^{\infty} \prod_{l=1}^{l} d \alpha_{i} e^{-i \Sigma \alpha_{2}(1-i \varepsilon)} \sum_{\mathscr{F}}^{\prime} \hat{D}_{G}^{\mathscr{F}}(\alpha, \varepsilon ; g) \\
& \times\left\{\prod_{F \in \mathscr{F}}\left(-t_{F}\right)\left[\frac{1}{U_{G}^{2}(\alpha)} \exp \left(-i \frac{V_{G}(\mathbf{p}, \alpha)}{U_{G}(\alpha)}(1-i \varepsilon)+i \frac{V_{G}\left(r^{2}, \omega, \alpha\right)}{U_{G}(\alpha)}\right)\right]\right\} \\
= & \int_{0}^{\infty} \ldots \int_{0}^{\infty} \prod_{i=1}^{l} d \alpha_{i} e^{-i \Sigma \alpha_{2}(1-i \varepsilon)} \sum_{\mathscr{F}}^{\prime} \hat{D}_{G}^{\mathscr{F}}(\alpha, \varepsilon ; g) \prod_{F \in \mathscr{F}}\left(-t_{F}\right) \\
& \times\left[\frac{1}{U_{G}^{2}(\alpha)} \exp \left(-i \frac{V_{G}(\mathbf{p}, \alpha)}{U_{G}(\alpha)}(1-i \varepsilon)\right) \int_{0}^{\infty} d r \varphi(r) \exp \left(i \frac{V_{G}\left(r^{2}, \omega, \alpha\right)}{U_{G}(\alpha)}\right)\right] .
\end{aligned}
$$


The interchange of the application of the $t_{F}$ operators and $\alpha$-integration on one side and $r$ integration on the other one is justified because the renormalized integrand is free from singularities for any subset of the $\alpha_{i}$ going to zero (renormalization), exponentially bounded for $\alpha_{i} \rightarrow \infty$ (mass term) and because as a function of $r$ it is bounded by a sum of polynomials with $\alpha$-dependent coefficients [7] so that the fall-off of $\varphi(r)(\mathrm{S} 2)$ !) is sufficient to ensure convergence. We thus may apply Fubini's theorem.

The mistrustful reader may again use the explicit (lengthy!) expression obtained on performing $\prod_{F \in \mathscr{F}} t_{F}[7$, Lemma III.4 (err.)] to get fully convinced.

Since $V_{G}\left(r^{2}, \omega, \alpha\right)$ and $U_{G}(\alpha)$ are both nonnegative functions for all values of $r^{2}$, $\omega, \alpha$ and $V_{G}\left(r^{2}, \omega, \alpha\right)$ has the form $V_{G}\left(r^{2}, \omega, \alpha\right)=\widetilde{V}(\omega, \alpha) r^{2}[\mathrm{cf} .(6)]$ we may rotate the $r$ integration path in (26) by $+\frac{\pi}{2}$. We have

$$
\int_{0}^{\infty} d r \varphi(r) \exp \left[i \frac{V_{G}\left(r^{2}, \omega, \alpha\right)}{U_{G}(\alpha)}\right]=i \int_{0}^{\infty} d r \varphi(i r) \exp \left[-i \frac{V_{G}\left(r^{2}, \omega, \alpha\right)}{U_{G}(\alpha)}\right] .
$$

With this expression we go back to (26) and interchange again $r$ integration and $t_{F}$ operation plus $\alpha$-integration. Now we can perform the Wick rotation in $\alpha$-parametric space by simultaneously rotating all $\alpha$-parameters according to $\alpha \rightarrow-i \alpha$. This may be realized by scaling the $\alpha$-parameters according to $\alpha \rightarrow \lambda \alpha$ and rotating $\lambda \rightarrow-i \lambda$. The procedure is precisely described in [17] for a diagram without renormalization parts and dressing factors. The latter do not cause any harm for the relevant $g$ [see (21) and the following remarks] and the former don't either: the renormalization operators $t_{F}$ generate products of expressions $\left(-i \frac{\widetilde{V}_{G}\left(p_{E u}^{2}\left(r^{2}, \omega, \mathbf{p}\right), \alpha\right)}{\widetilde{U}_{G}(\alpha)}\right)$ (where ${ }^{\sim}$ indicates that the summation in $(6)$ is restricted to certain subsets of one- or two-trees) with good homogeneity properties: they pick up a factor $(-i)$ on transforming $\lambda \rightarrow(-i \lambda)$ which together with the $(-i)$ in front gives just the minus sign of the Euclidean exponent (5). The $(-i)$ in front and the Euclidean squares in $\widetilde{V}$ appear as a consequence of the previous Wick rotation in momentum space (27). We arrive at the following result:

$$
\left.\lim _{\varepsilon \rightarrow 0} \int_{0}^{\infty} d r I_{G, \varepsilon}\left(r^{2}, \omega\right), \mathbf{p} ; g\right) \varphi(r)=i^{E} \int_{0}^{+2} d r I_{G, d r}\left(r^{2}, \omega, \mathbf{p} ; g\right) \varphi(i r)
$$

(the factor $i^{\frac{E}{2}+1}$ is a remnant of the $\alpha$-Wick rotation [17]). At this stage we could already apply the Euclidean results R1) to R3) to estimate (28) in terms of the bound on the sum over dressed Euclidean amplitudes and $\int d r|\varphi(i r)|$. But before doing so we want to provide some additional information on this integral and then apply some basic results from functional analysis to enlarge the domain of the functional

$$
\left.\lim _{\varepsilon \rightarrow 0} \int d r I_{G, \varepsilon}\left(r^{2}, \omega\right), \mathbf{p} ; g\right) \ldots
$$

and to put it in a setting more easily accessible to further mathematical studies.

From a physical point of view the test function has to model the spread of the external momenta over a certain range for some given process. So we are especially 
interested in the case where the momenta are concentrated around a possibly rather sharp peak. Since $S(23)$ is dense in $L^{2}$ we can certainly approximate such a situation within $S$. A simple choice of a class of test functions is

$$
\varphi_{\alpha A C}^{(\delta)}(r)=C \exp \left\{-\alpha\left(r+\frac{A^{2}}{r}\right)\right\} e^{i \delta r} ; \quad C, \alpha, A, \delta \in \mathbb{R} ; \alpha, \delta>0 .
$$

These functions have their maxima at $r=A$ and are sharply peaked there for large $\alpha$. For $\delta \ll \alpha$ they are approximately real too, but for $\delta=0$ they do not belong to $S$ any more and $\int|\varphi(i r)| d r$ does not exist. We shall see in a moment, however, that it is possible to extend (28) to functions e.g.

$$
\tilde{\varphi}_{\alpha A C}(r)=f(r) \varphi_{\alpha A C}^{(0)}(r)
$$

where $f(r)$ fulfills S1) and

$$
\int\left|f\left(e^{i \gamma} r\right)\right| d r<\infty, \quad 0 \leqq \gamma \leqq \frac{\pi}{2},
$$

so that we can also approximate the peak e.g. by

$$
\tilde{\varphi}_{\alpha A C}(r)=\frac{2 A}{(r+A)^{2}} \varphi_{\alpha A C}^{(0)}(r)
$$

which is real and still normalizable after Wick rotation $r \rightarrow i r$. For $\alpha A \gg 1$ we have

$$
\int_{0}^{\infty}\left|\tilde{\varphi}_{\alpha A C}(r)\right| d r=C K_{1}(2 A \alpha)\left(1+O\left(\frac{1}{\alpha A}\right)\right)=C\left(\frac{\pi}{2 A \alpha}\right)^{1 / 2} e^{-2 A \alpha}\left(1+O\left(\frac{1}{\alpha A}\right)\right)
$$

( $K_{1}$ is a modified Bessel function of the second kind)

$$
\int_{0}^{\infty}\left|\tilde{\varphi}_{\alpha A C}(i r)\right| d r=\pi C,
$$

so that the $\left(L^{1}\right)$-norm of $\tilde{\varphi}$ grows exponentially in $\alpha A$ on Wick rotation. Similarly if we approximate the peak by

$$
\sim \frac{r^{n}}{(r+A)^{2 n}}
$$

the blow up factor is found to be proportional to $2^{n}$. Better estimates for $\int I_{G, \varepsilon} \ldots$ cannot be expected as long as we only make use of the general analyticity properties of Feynman amplitudes.

We now want to clarify the structure of (28) from the point of view of functional analysis. To do so we introduce the linear spaces

$$
S_{ \pm}=\left\{\varphi_{ \pm} \mid \varphi_{ \pm}:[0, \infty) \rightarrow \mathbb{C}, \varphi_{ \pm} \text {fulfills S1), S2), S3+) respectively S3-) }\right\},(31)
$$

where S1), S2) were given before and

$$
\mathrm{S} 3 \pm) \quad \varphi_{ \pm}\left(\frac{i}{r}\right)= \pm \frac{r^{2}}{i} \varphi_{ \pm}(r) .
$$

We have

Lemma 2. i) $S_{+}+S_{-}$is dense in $L_{1}^{2}$ (and thus in any $L_{\alpha}^{p}$ ), where

$$
L_{\alpha}^{p}=L^{p}\left([0, \infty), r^{\alpha} d r\right), \quad \alpha \in \mathbb{R} .
$$


ii) $\widetilde{S}_{ \pm} \perp \widetilde{S}_{+}^{*}, L_{1}^{2} \cong \widetilde{S}_{ \pm}+\widetilde{S}_{+}^{*}$, where $\widetilde{S}_{ \pm}=\overline{\left(S_{ \pm} \cap L_{1}^{2}\right)}$, i.e. the closure of $S_{ \pm}$with respect to the norm topology of $L_{1}^{2}$, and $S^{*}:=\left\{\varphi \mid \varphi^{*} \in S\right\}$.

The proof of Lemma 2 is given in the appendix. Part ii) is not necessary for the following. It is only included because it gives some insight into the structure of the spaces involved.

For $\varphi_{ \pm} \in S_{ \pm}$we immediately get from (28)

$$
\lim _{\varepsilon \rightarrow 0} \int_{0}^{\infty} d r I_{G, \varepsilon}\left(r^{2}, \omega, \mathbf{p} ; g\right) \varphi_{ \pm}(r)= \pm i^{\frac{E}{2}+1} \int_{0}^{\infty} d r I_{G, d r}\left(\frac{1}{r^{2}}, \omega, \mathbf{p} ; g\right) \varphi_{ \pm}(r)
$$

on transforming $r \rightarrow \frac{1}{r}$.

Due to the uniform bounds on $I_{G, d r}$ this implies that on the spaces $S_{ \pm} C L_{0}^{1}$ the Minkowski space functional is bounded by the $L^{\infty}$ (sup-)norm of the Euclidean amplitude. Lemma 2, ii) says $S_{+}$and $S_{-}$span (roughly speaking) one half of the Hilbert space $L_{1}^{2}$. Obviously it cannot be possible to approximate a $\delta$-peak within $S_{ \pm}$. It is only possible to approximate the peak by taking the modulus of functions which are in $S_{ \pm}$as seen from the examples

$$
\begin{aligned}
& \frac{1}{r^{2}+i} \exp \left[\alpha\left\{-\left(r+\frac{1}{r}\right)+i\left(r-\frac{1}{r}\right)\right\}\right] \in S_{+}, \\
& \frac{r^{2}-i}{\left(r^{2}+i\right)^{2}} \exp \left[\alpha\left\{-\left(r+\frac{1}{r}\right)+i\left(r-\frac{1}{r}\right)\right\}\right] \in S_{-},
\end{aligned}
$$

the moduli of which are peaked at $r=1$. Peaks at $r=A$ can be realized by functions fulfilling

$$
\varphi_{ \pm}^{(A)}\left(\frac{i A^{2}}{r}\right)= \pm \frac{r^{2}}{i A^{2}} \varphi_{ \pm}^{(A)}(r)
$$

which also fulfill (32).

We want to enlarge the space of functions on which $\lim _{\varepsilon \rightarrow 0} \int I_{G, \varepsilon} \ldots$ is well-defined.

As a consequence of (14) and R3) both sides of (28) are well-defined for

$$
\varphi_{ \pm} \in \overline{S_{ \pm} \cap L_{0}^{1}}=: S_{0 \pm}^{1} \text { for } E>2
$$

and for $\varphi_{ \pm} \in \overline{S_{ \pm} \cap L_{2}^{1} \cap L_{-2}^{1}}=: S_{2 \pm}^{1}$ for $E=2$ (i.e. a contribution to the two-point function). In the last case closure is intended with respect to the norm $\int_{0}^{\infty} d r\left(\frac{1}{r^{2}}+r^{2}\right)|\varphi(r)|$. On noticing that $\int I_{G, \varepsilon}, \ldots, \int I_{G, d r} \ldots$ operate continuously on $S_{0 \pm}^{1}$ respectively $S_{2 \pm}^{1}$ (28) still has to be true there as well as on the spaces

$$
S_{0}=S_{0+}^{1}+S_{0-}^{1}, \quad(E>2), \quad S_{2}:=S_{2+}^{1}+S_{2-}^{1}, \quad(E=2) .
$$

Finally we define the vector spaces with norm

$$
\begin{aligned}
B_{0 / 2} & =\left(S_{0 / 2},\|\|_{0 / 2}\right), \\
\|\varphi\|_{0 / 2}: & =\int d r\left(\left|\varphi_{+}(r)\right|+\left|\varphi_{-}(r)\right|\right)\left(\frac{1}{r^{2}}+r^{2}\right)^{\frac{1}{2}(0 / 2)} .
\end{aligned}
$$


For $\varphi \in B_{0 / 2}$ we now may perform the sum over dressed Feynman amplitudes. Summing over all planar graphs with $E$ external legs and the number of vertices varying between $n_{1}$ and $n_{2}$, we get

$$
\begin{aligned}
& \mid \sum_{G_{E}}: n(G)=n_{1} \\
& \quad=\left|\sum_{n_{1}}^{n_{2}} g^{n(G)} \lim _{\varepsilon \rightarrow 0} \int d r I_{G, \varepsilon}\left(r^{2}, \omega, \mathbf{p} ; g\right) \varphi(r)\right| \\
& \quad \leqq K \sum_{n_{1}}^{n_{2}}\left(C K_{E}(\eta)\right)^{n} \int d r\left(\left|\varphi_{+}(r)\right|+\left|\varphi_{-}(r)\right|\right)\left(\frac{1}{r^{2}}+\mathbf{p}^{2}\right)^{\delta_{E, 2}} \\
& \quad \leqq K \sum_{n_{1}}^{n_{2}}\left(C K_{E}(\eta)\right)^{n}\left(\mathbf{p}^{2}+1\right)^{\delta_{E, 2}}\|\varphi\|_{0 / 2} .
\end{aligned}
$$

Here we used the geometric bound on the number of planar graphs (2) and R3). The last estimate implies the series to be uniformly Cauchy for $\operatorname{Re} g>0,|g|<\eta(\mathrm{R} 3))$ and thus convergent. We arrive at the following results:

RM1: The sum over all dressed amplitudes of 1PI planar $\varphi_{4}^{4}$ graphs with $E$ external legs for $\operatorname{Re} g>0,|g|<\eta, \eta$ sufficiently small (s.s.) uniquely defines a family of bounded linear functionals $F_{g \omega \mathbf{p}}^{(E)}$ on the Banach spaces $B_{0}(E>2)$ or $B_{2}(E=2)(35)$ via

$$
F_{g() \mathbf{p}}^{(E)}(\varphi)=\lim _{\varepsilon \rightarrow 0} \sum_{G_{E}}^{\infty} \sum_{n(G)=0}^{\infty} g^{n(G)} \int_{0}^{\infty} d r I_{G, \varepsilon}\left(r^{2}, \omega, \mathbf{p} ; g\right) \varphi(r) .
$$

Their norm is bounded by

$$
\left\|F_{g \omega \mathbf{p}}^{(E)}\right\| \leqq K \sum_{n=0}^{\infty}\left|C K_{E}(\eta)\right|^{n}\left(1+\mathbf{p}^{2}\right)^{\delta_{E, 2}}
$$

i.e. by the bound on the Euclidean sum. Alternatively $F_{g \omega \mathbf{p}}^{(E)}$ may be looked upon as an unbounded densely defined linear functional on

$$
L^{1}\left([0, \infty),\left(r^{2}+\frac{1}{r^{2}}\right)^{\delta_{E, 2}} d r\right)
$$

with domain $S_{0}(E>2)$ or $S_{2}(E=2)(34)$. It is then bounded on the subspaces $S_{0 \pm}^{1}$ or $S_{2 \pm}^{1}$.

RM2: (Borel summability). There exists a constant $K$ such that for $\operatorname{Re} g>0,|g|<\eta$ s.s. one has for any $k \in \mathbb{N}$ and $\varphi \in B_{0 / 2}$,

$$
\begin{aligned}
& \left|F_{g \omega \mathbf{p}}^{(E)}(\varphi)-\sum_{G / n(G) \leqq k-1} g^{n(G)} \lim _{\varepsilon \rightarrow 0} \int_{0}^{\infty} d r I_{G, \varepsilon}^{u d}\left(r^{2}, \omega, \mathbf{p}\right) \varphi(r)\right| \\
& \leqq k !(K|g|)^{k}\|\varphi\|_{0 / 2}\left(1+\mathbf{p}^{2}\right)^{\delta_{E, 2}},
\end{aligned}
$$

where the sum is over the undressed amplitudes $I_{G, \varepsilon}^{u d}$ (without $\hat{D}_{G}^{\mathscr{F}}$ factors) in Minkowski space of all graphs $G$ with $E$ external legs and less than $k$ vertices. 
RM3: For any graph $G$ of order $n$, planar or not, and $\operatorname{Reg}>0,|g|<\eta$ s.s. and $\varphi \in B_{0 / 2}$, we have the estimate

$$
\left|\lim _{\varepsilon \rightarrow 0} g^{n(G)} \int_{0}^{\infty} d r I_{G, \varepsilon}\left(r^{2}, \omega, \mathbf{p} ; g\right) \varphi(r)\right| \leqq K_{E}^{n(G)}(\eta)\|\varphi\|_{0 / 2}\left(1+\mathbf{p}^{2}\right)^{\delta_{E, 2}} .
$$

On the same reasoning as in the Euclidean case and on making use of the remarks following (21) one may - eventually at the cost of a smaller $\eta$ - replace $\operatorname{Re} g>0$ by $|\arg g|<\pi-\delta$ for any $\delta>0$, where $\eta \rightarrow 0$ for $\delta \rightarrow 0$.

\section{Summary and Discussion}

Rivasseau's results implying the Borel summability of planar $\varphi_{4}^{4}$ have been transmitted to Minkowski space. Minkowski space sums of dressed Green functions can be interpreted as bounded linear functionals on a Banach space. Bounds on Euclidean quantities turn into bounds on the norms of those functionals. From a general mathematical point of view their domain is as large as can be expected, namely it is dense in $L^{1}$ or $L^{2}$ spaces on the external momentum variables with respect to the norm-topology. The functionals could not be defined as tempered distributions, however, which would have been nice in view of Wightman's axioms. This would require a more detailed knowledge of all possible Feynman diagram singularities. Our construction is certainly not unique in the sense that we could have started from different domains for the functionals. So we could have replaced $\mathrm{S} 3 \pm$ ) Sect. III by

$$
\varphi_{ \pm}\left(\begin{array}{c}
i \\
r
\end{array}\right)= \pm \varphi_{ \pm}(r)
$$

or something similar [see also (33)] without major consequences. But it is not possible to replace the involution $r \rightarrow \frac{i}{r}$ by e.g. $r \rightarrow i r$ which leads to conflicts between the required analyticity and fall-off properties of $\varphi$ and the global behaviour of holomorphic functions, e.g. Liouville's theorem. The invariance conditions $\mathrm{S} 3 \pm$ were not necessary to perform the Wick-rotation. They only show that there are closed subspaces $S_{0 / 2+}^{1}$ and $S_{0 / 2-}^{1}$ - spanning together the whole domain - on which the functionals are bounded also with respect to a $L^{1}$ norm.

One may look for invariance conditions of the form

$$
\varphi(i r)= \pm(T \varphi)(r),
$$

where $T$ is a suitable bounded operator, a simple choice being $T \varphi=\varphi^{*}$. In this case we would get the $+(-)$ sign for any

$$
\varphi(r)=P\left(\frac{1-i}{2} r\right) e^{-\frac{1-i}{2} r},
$$

where $P$ is some polynomial with real (purely imaginary) coefficients. Those $\varphi$ together form a dense set in $L^{2}$, but the invariant sets are not linear with respect to $\mathbb{C}$. Certainly there is no analogue of Lemma $2 \mathrm{ii}$ ). 
Quite generally the knowledge on the Minkowski space quantities and also on their Euclidean counterparts, if to a smaller extent, remains very coarse. One way along which one might try to extract additional information is to make use of the fact that $r=0$, i.e. all external momenta spacelike, always is an inner point of the Euclidean domain of any Feynman diagram in a massive theory. With the help of majorization techniques, see e.g. [18] and perhaps making use of planarity one might extract some information on the Borel sum in that region.

A characterisation of the singularity structure in momentum space of summed amplitudes seems to be a far more difficult problem.

\section{Appendix}

We want to prove Lemma 2 Sect. III and make some further comments on the spaces $\widetilde{S}_{ \pm}$.

Proof of $i$ ). Let

$$
K_{ \pm}=\left\{\varphi_{ \pm}(r) \mid \varphi_{ \pm}:[0, \infty) \rightarrow \mathbb{C}, \varphi_{ \pm}(r)=\psi_{ \pm}(r) w_{0}(r)\right\},
$$

where $\psi_{ \pm}(r)$ have to fulfill S1), S3 \pm$)$ Sect. III and $\psi_{ \pm}\left(r e^{i \gamma}\right)$ is uniformly bounded for $0 \leqq r<\infty, 0 \leqq \gamma \leqq \frac{\pi}{2}$, (A 1 )

$$
w_{0}(r):=\exp \left\{-\left(r+\frac{1}{r}\right)+i\left(r-\frac{1}{r}\right)\right\}
$$

then $\psi_{ \pm}(r) w_{0}(r) \in S_{ \pm}$.

Therefore

$$
K_{+}+K_{-} \subset S_{+}+S_{-} C L_{1}^{2}
$$

$K_{+}+K_{-}$contains all functions of the form $\varphi(r)=\psi(r) w_{0}(r)$, where $\psi(r)$ fulfills S1) and (A1).

The density of $K_{+}+K_{-}$in $L_{1}^{2}$ is equivalent to the triviality of its orthogonal complement in $L_{1}^{2}$. Thus assume $(g, \varphi):=\int_{0}^{\infty} d r r g^{*}(r) \varphi(r)=0$ for some $g \in L_{1}^{2}$ and all $\varphi \in K_{+}+K_{-}$. Then

$$
(\tilde{g}, \tilde{\varphi})=0 \quad \text { for } \quad \tilde{g}(r)=g(r) \exp \left\{-i\left(r-\frac{1}{r}\right)\right\}
$$

and

$$
\tilde{\varphi}(r)=\varphi(r) \exp \left\{-i\left(r-\frac{1}{r}\right)\right\} .
$$

We now make a choice for $\psi(r)$. Let

$$
\psi_{n}(r)=\frac{1}{(r+1)^{n}}, \quad \tilde{\varphi}_{n}(r)=\psi_{n}(r) \exp \left\{-\left(r+\frac{1}{r}\right)\right\}, \quad n \in \mathbb{N} .
$$


Then we have for all $n \in \mathbb{N}$ on assumption

$$
0=\left(\tilde{g}, \tilde{\varphi}_{n}\right)=\int_{0}^{\infty} d r r \tilde{g}^{*}(r) \tilde{\varphi}(r)=\int_{0}^{1} d x(1-x) \tilde{g}^{*}\left(\frac{1-x}{x}\right) x^{n-3} \exp \left\{-\frac{x^{2}+(1-x)^{2}}{x(1-x)}-\right\},
$$

which on the completeness of the polynomials $x^{n}$ on $[0,1]$ with the weight

$$
(1-x) \exp \left\{-\frac{x^{2}+(1-x)^{2}}{x(1-x)}\right\}
$$

strictly positive in $(0,1)$, implies $\tilde{g}^{*}\left(\frac{1-x}{x}\right) \cong 0$ in $L^{2}([0,1], d x)$, therefore $\tilde{g}(r) \cong 0$ and $g(r) \cong 0$ in $L_{1}^{2}$.

Proof of ii). Orthogonality of $\widetilde{S}_{ \pm}$and $\widetilde{S}_{ \pm}^{*}$ : let $\varphi_{ \pm} \in S_{ \pm} \cap L_{1}^{2}$,

$$
\begin{aligned}
\left(\varphi_{\mp}^{*}, \varphi_{ \pm}\right): & =\int_{0}^{\infty} d r r \varphi_{\mp}(r) \varphi_{ \pm}(r)=i^{2} \int_{0}^{\infty} d r r \varphi_{\mp}(i r) \varphi_{ \pm}(i r) \\
& =i^{2} \int_{0}^{\infty} d r \frac{1}{r^{3}} \varphi_{\mp}\left(\frac{i}{r}\right) \varphi_{ \pm}\left(\frac{i}{r}\right)=-\int_{0}^{\infty} d r r \varphi_{\mp}(r) \varphi_{ \pm}(r)=-\left(\varphi_{+}^{*}, \varphi_{ \pm}\right),
\end{aligned}
$$

where S1), S2), S3 \pm ) have been used. The orthogonality of $S_{+}$and $S_{-}^{*}$ implies the one of $\widetilde{S}_{+}$and $\tilde{S}_{-}^{*}$.

Completeness of $\widetilde{S}_{+}+\widetilde{S}_{-}^{*}$ in $L_{1}^{2}$ is equivalent to its orthogonal complement consisting of 0 only.

We choose $\varphi_{n}^{+}(r)=a_{0}(r) e^{i 2 n \alpha(r)}, n \in \mathbb{N}_{0}$, where

$$
a_{0}(r)=\frac{1}{r^{2}+i}, \quad e^{i n \alpha(r)}=\left(\frac{r^{2}-i}{r^{2}+i}\right)^{n},
$$

so that $\varphi_{n}^{+}(r) \in \widetilde{S}_{+}$; and we choose $\varphi_{n}^{-*}(r)=a_{0}^{*}(r) e^{-i(2 n+1) \alpha(r)}, n \in \mathbb{N}_{0}$ so that $\varphi_{n}^{-*}(r) \in \widetilde{S}_{-}^{*}$. Assume

$$
\int_{0}^{\infty} d r r \varphi_{n}^{+}(r) g(r)=\int_{0}^{\infty} d r r \varphi_{n}^{-*}(r) g(r)=0
$$

for some $g(r) \in L_{1}^{2}$ and all $n \in \mathbb{N}_{0}$.

We have $\alpha(r)=\arccos \left(\frac{r^{4}-1}{r^{4}+1}\right)$ varying strictly monotonically from $-\pi$ to 0 for $r$ between 0 and $\infty$. Therefore

$$
\int_{0}^{\infty} \varphi_{n}^{+}(r) g(r) d r=\frac{1}{2} \int_{-2 \pi}^{0} a_{0}\left(\alpha^{-1}\left(\frac{x}{2}\right)\right) g\left(\alpha^{-1}\left(\frac{x}{2}\right)\right) e^{i n x} \frac{d x}{\alpha^{\prime}\left(\alpha^{-1}\left(\frac{x}{2}\right)\right)} \quad(x=2 r)
$$

and

$$
\begin{aligned}
\int_{0}^{\infty} \varphi_{n}^{-*}(r) g(r) d r & =\int_{0}^{\infty} a_{0}^{*}(r) e^{-i(2 n+1) \alpha(r)} g(r) d r=\int_{0}^{\infty} a_{0}(r) e^{-i(2 n+2) \alpha(r)} g(r) d r \\
& =\frac{1}{2} \int_{-2 \pi}^{0} a_{0}\left(\alpha^{-1}\left(\frac{x}{2}\right)\right) g\left(\alpha^{-1}\left(\frac{x}{2}\right)\right) e^{-i(n+1) x} \frac{d x}{\alpha^{\prime}\left(\alpha^{-1}\left(\frac{x}{2}\right)\right)} .
\end{aligned}
$$


Thus from the completeness of $\left\{e^{i n x}, n \in \mathbb{Z}\right\}$ in $L^{2}([-2 \pi, 0], d x)$

$$
a_{0}\left(\alpha^{-1}\left(\frac{x}{2}\right)\right) g\left(\alpha^{-1}\left(\frac{x}{2}\right)\right) \frac{1}{\alpha^{\prime}\left(\alpha^{-1}\left(\frac{x}{2}\right)\right)} \cong 0
$$

in $L^{2}([-2 \pi, 0], d x)$ and therefore $g(r) \cong 0$ in $L_{1}^{2}$.

The same procedure is possible for $S_{-}+S_{+}^{*}$. q.e.d.

In Sect. III the test functions have been split into parts $\varphi_{ \pm} \in S_{ \pm}$. Contrary to $\widetilde{S}_{+}$ and $\widetilde{S}_{-}^{*}, \widetilde{S}_{+}$and $\widetilde{S}_{-}$are not orthogonal. In fact the angle between two unit vectors in $\widetilde{S}_{+}$and $\widetilde{S}_{-}$may become arbitrarily small. As can be seen from the previous proof, the situation is quite similar to the subspaces $\left\{\sum_{n} a_{n} e^{i n x}, n \in \mathbb{N}_{0}\right\}$ and $\left\{\sum_{n} a_{n} e^{i\left(n+\frac{1}{2}\right) x}, n \in \mathbb{N}_{0}\right\},\left(\sum\left|a_{n}\right|^{2}<\infty\right)$ of $L^{2}([0,2 \pi], d x)$. Thus it is not possible to estimate $\left\|\varphi_{+}\right\|_{L_{1}^{2}},\left\|\varphi_{-}\right\|_{L_{1}^{2}}$ in terms of $\|\varphi\|_{L_{1}^{2}}$ for a general $\varphi=\varphi_{+}+\varphi_{-}$. This would be sufficient [see (32)] to make $F_{g \omega \text { p }}$ (essentially) a bounded functional on $L^{2}$. Splitting up into $\tilde{S}_{+}$and $\widetilde{S}_{-}^{*}$ does not help either because functions of $\tilde{S}^{*}$ have no invariance properties $\mathrm{S} 3 \pm$ ) (and possibly singularities in the upper right half plane).

Acknowledgements. I am indebted to Prof. G. 't Hooft for proposing the subject, for discussions and valuable comments on a preliminary version of the paper, to Prof. V. Rivasseau for explanatory correspondence on his work.

\section{References}

1. 't Hooft, G.: On the convergence of a planar diagram expansion. Commun. Math. Phys. 86, 449 (1982)

2. 't Hooft, G.: Rigorous construction of planar diagram field theories in four-dimensional Euclidean space. Commun. Math. Phys. 88, 1 (1983)

3. Rivasseau, V.: Construction and Borel summability of planar 4-dimensional Euclidean field theory. Commun. Math. Phys. 95, 445 (1984)

4. Coleman, S.: $1 / N$. Erice lectures 1979. Zichichi, A. (ed.)

5. Osterwalder, K., Schrader, R.: Axioms for Euclidean Green functions. Commun. Math. Phys. 31, 83 (1973) and Axioms for Euclidean Green functions. II. Commun. Math. Phys. 42, 281 (1975)

6. Bardeen, W.A., Moshe, M.: Phase structure of the $O(N)$ vector model. Phys. Rev. D 28, 1372 (1983)

7. de Calan, C., Rivasseau, V.: Local existence of the Borel transform in Euclidean $\varphi_{4}^{4}$. Commun. Math. Phys. 82, 69 (1981); Commun. Math. Phys. 91, 265 (1983) (Erratum)

8. 't Hooft, G.: A planar diagram theory for strong interactions. Nucl. Phys. B 72, 461 (1974)

9. Koplik, J., Neveu, A., Nussinov, S.: Some aspects of the planar perturbation series. Nucl. Phys. B 123, 109 (1977)

10. Lautrup, B.: On high order estimates in QED. Phys. Lett. 69B, 109 (1977)

11. 't Hooft, G.: Can we make sense out of "quantum chromodynamics?". Erice Lectures 1977. Zichichi, A. (ed.)

12. Parisi, G.: Singularities of the Borel transform in renormalizable theories. Phys. Lett. $76 \mathrm{~B}, 65$ (1978), and The Borel transform and the renormalization group. Phys. Rep. 49, 215 (1979)

13. Zimmermann, W.: Convergence of Bogoliubov's method for renormalization in momentum space. Commun. Math. Phys. 15, 208 (1969) 
14. Bergère, M., Zuber, J.B.: Renormalization of Feynman amplitudes and parametric integral representation. Commun. Math. Phys. 35, 113 (1974); Bergère, M., Lam, Y.M.P.: BogoliubovParasiuk theorem in the $\alpha$-parametric representation. J. Math. Phys. 17, 1546 (1976)

15. Nakanishi, N.: Graph theory and Feynman integrals. New York: Gordon and Breach 1970

16. Zimmermann, W.: The power counting theorem for Minkowski metric. Commun. Math. Phys. 11, 1 (1968)

17. Itzykson, C., Zuber, J.-B.: Quantum field theory. Chap. 6-2-4. New York: McGraw Hill 1980

18. Todorov, I.T.: Analytic properties of Feynman diagrams in quantum field theory. Oxford: Pergamon Press 1966

Communicated by K. Osterwalder

Received May 5, 1987; in revised form September 4, 1987 